\title{
LC-MS Differential Analysis for Fast and Sensitive Determination of Biotransformation of Therapeutic Proteins ${ }^{\llbracket}$
}

\author{
Ming Yao, ${ }^{1}$ Bingming Chen, ${ }^{1}$ Weiping Zhao, John T. Mehl, Lingjun Li, and Mingshe Zhu \\ Pharmaceutical Candidate Optimization, Bristol-Myers Squibb, Princeton, New Jersey (M.Y., W.Z., J.T.M., M.Z.); \\ School of Pharmacy and Department of Chemistry, University of Wisconsin-Madison, Madison, Wisconsin (B.C., L.L.); \\ School of Life Sciences, Tianjin University, Nankai, Tianjin, People's Republic of China (L.L.); and MassDefect
} Technologies, Princeton, New Jersey (M.Z.)

Received July 28, 2017; accepted January 29, 2018

\begin{abstract}
Therapeutic biologics have become a fast-growing segment within the pharmaceutical industry during the past 3 decades. Although the metabolism of biologics is more predictable than small molecule drugs, biotransformation can significantly affect the activity of biologics. Unfortunately, there are only a limited number of published studies on the biotransformation of biologics, most of which are focused on one or a few types of modifications. In this study, an untargeted LC-MS-based differential analysis approach was developed to rapidly and precisely determine the universal biotransformation profile of biologics with the assistance of bioinformatic tools. A
\end{abstract}

human monoclonal antibody (mAb) was treated with $t$-butyl hydroperoxide and compared with control mAb using a bottom-up proteomics approach. Thirty-seven types of post-translational modifications were identified, and 38 peptides were significantly changed. Moreover, although all modifications were screened and detected, only the ones related to the treatment process were revealed by differential analysis. Other modifications that coexist in both groups were filtered out. This novel analytical strategy can be effectively applied to study biotransformation-mediated protein modifications, which will streamline the process of biologic drug discovery and development.

\section{Introduction}

Since the approval and commercialization of the first monoclonal antibody (mAb) in 1986, the development of therapeutic biologics has captured significant attention within the pharmaceutical industry during the past 3 decades (Waldmann, 2003; Xu and Vugmeyster, 2012). In general, the term "therapeutic biologics" refers to a substance derived from living organisms that is applicable to prevent, treat, or cure a human disease or condition (Hamuro and Kishnani, 2012; Zhao et al., 2012). In addition to mAbs, which represent the majority of marketed biologics, other biologic modalities, such as therapeutic peptides, bifunctional biologics, and antibody-drug conjugates (ADCs), have rapidly emerged ( $\mathrm{Xu}$ and Vugmeyster, 2012). Some therapeutic proteins contain artificial amino acids, unique peptide sequences or linkage structures, or small-molecule drug moieties (Hamuro and Kishnani, 2012; Katsila et al., 2012; Xu and Vugmeyster, 2012).

Like endogenous proteins, antibody therapeutics undergo catabolism, such as oxidation, deamination, glycosylation, phosphorylation, and

${ }^{1}$ M.Y. and B.C. contributed equally to this work.

L.L. received financial support from the National Institutes of Health [Grant R56$\mathrm{MH}-110215]$, a Vilas Distinguished Achievement Professorship, and a Janis Apinis Professorship with funding provided by the Wisconsin Alumni Research Foundation and University of Wisconsin-Madison School of Pharmacy. No potential conflicts of interest relevant to this article were reported.

https://doi.org/10.1124/dmd.117.077792.

S This article has supplemental material available at dmd.aspetjournals.org. isomerization, in a biologic system to form catabolites (peptides and amino acids). Here we define biotransformation of a therapeutic protein as a chemical or enzymatic process occurring within a biologic system in vitro or in vivo that changes the structure of the therapeutic protein. Biotransformation of a therapeutic protein can follow either predictable catabolism pathways, similar to the disposition and elimination processes of endogenous proteins, or pathways different from protein catabolism due to the presence of an artificial amino acid, peptide sequence, linkage structure of amino acids, and conjugated drug (Cheloha et al., 2014; Walles et al., 2016; Zhang et al., 2016). Biotransformation-derived protein modifications may have a profound impact on the activity or disposition and clearance behavior of the biologics, as they can potentially lead to reduced target-binding efficiency, shorter halflife, higher clearance rate, poor bioavailability, increased off-target binding/toxicity, increased immunogenicity, and altered assay detection (Liu et al., 2009, 2011; Cai et al., 2011; Wang et al., 2011; Hall, 2014). For example, the methionine oxidation in human IgG1 causes significant reduction in binding affinity and reduced serum half-life (Wang et al., 2011); the isomerization of a single Asp residue leads to complete loss of target binding in a model $\mathrm{mAb}$ (mAb X) (Prueksaritanont and Tang, 2012).

Usually, drug metabolism and pharmacokinetic research of therapeutic proteins relies on ligand-binding assays (LBAs), such as enzyme-linked immunosorbent assay, for protein quantitative analysis during drug development. LBAs can be highly sensitive, are typically easy to operate, and are capable of high-throughput analysis. Recently, quantitative LC-MS-based methods have

ABBREVIATIONS: ADC, antibody-drug conjugate; AMBIC, ammonium bicarbonate; DTT, dithiothreitol; FA, formic acid; HRMS, high-resolution mass spectrometry; IDA, information-dependent acquisition; LBA, ligand-binding assay; LC, liquid chromatography; mAb, monoclonal antibody; MS, mass spectrometry; MS/MS, tandem mass spectrometry; m/z, mass/charge ratio; NQ, deamidation; PBS, phosphate-buffered saline; tBHP, $t$ butyl hydroperoxide; Zwitt, Zwittergent 3-12 detergent. 
increasingly been employed to support pharmacokinetic characterization during drug discovery and preclinical development (An et al., 2014). However, there is no effective methodology available to study global changes in biologics upon biotransformation, and most of the published biotransformation studies focus on only one type of modification (Liu et al., 2011; Wang et al., 2011; Bults et al., 2016; Kullolli et al., 2017). For example, Bults et al. (2016) reported an LC-MS approach to analyze trastuzumab and its NQ product in vivo, in which a selected reaction monitoring approach was used to monitor five molecular species from tryptic-digested trastuzumab from human plasma without enrichment. This method is highly specific to monitor the NQ at Asp55, and it is not a means to monitor global biotransformation of a therapeutic protein.

The investigation of therapeutic protein biotransformation relies on high-resolution MS (HRMS)-based proteomics workflows, such as a top-down approach, a bottom-up approach, and a combination of both approaches (Domon and Aebersold, 2006). Intact protein or protein assemblies are directly analyzed in a topdown proteomics workflow, which requires ultra-high-resolving powers and usually has limited protein sequence coverage and sensitivity (Ruan et al., 2001; Kang et al., 2017). In a typical bottom-up proteomics workflow, protein samples are enzymatically digested into peptides, which are then subjected to LC-MS experiments. The resulting tandem MS (MS/MS) spectra are compared with the protein sequence database to find the best matched peptide. To search for modified peptides, MS/MS spectra are compared with a list of predefined modifications on peptides in traditional database software. Some software packages, such as PEAKS (Bioinformatics Solutions Inc., Waterloo, ON, Canada), used an improved algorithm for PTM search that is capable of screening more than 650 known PTM types in one run (Han et al., 2011). Compared with the top-down workflow, a bottom-up approach offers several advantages, including improved sequence coverage, and superior analytical specificity, sensitivity, precision, and accuracy (Ewles and Goodwin, 2011; Xu and Vugmeyster, 2012; Kullolli et al., 2017).

A key analytical challenge in determining global biotransformation of therapeutic proteins via a bottom-up workflow is the large number of low-level protein modifications that are not associated with the biotransformation process. These modifications are either present in a dosing solution or are formed during a sample preparation, such as enzymatic hydrolysis, isolation, and enrichment processes. It would require significant effort and time to detect and characterize these modified proteins and to distinguish them from biotransformation-derived protein modifications. Herein, we developed and validated an untargeted LC-HRMS-based differential analysis method for rapid detection and identification of modified peptides derived from the biotransformation of therapeutic mAbs. A human mAb was forced oxidized and compared with an untreated control $\mathrm{mAb}$ using a bottom-up proteomics workflow. Generated LC-MS datasets were processed using bioinformatic tools for differential analysis. Modified peptides that had undergone significantly higher levels of biotransformation were highlighted and structurally characterized. Moreover, relative quantification of biotransformation-related modified peptides was performed. To our knowledge, this is the first study that analyzes the global biotransformation of mAbs using LC-HRMS and differential analysis. With relatively easy sample preparation and rapid data acquisition, this method provides a template for the drug discovery to characterize therapeutic biologics in a high-throughput manner.

\section{Materials and Methods}

Materials and Chemicals. All reagents were used without additional purifications. Mouse anti-human IgG R10Z8E9 was available in-house and Dynabeads (M-280 tosylactivated) was purchased from Invitrogen (Carlsbad, CA). Male rat serum was purchased from Bioreclamation IVT (New York, NY). Trypsin, used for $\mathrm{mAb}$ digestion, was purchased from Promega (Madison, WI). The phosphate-buffered saline (PBS) and all solvents used in LC mobile phases (water, acetonitrile, and formic acid (FA) were purchased from GE Healthcare Bio-Sciences (Marlborough, MA). $t$-Butyl hydroperoxide (tBHP), dithiothreitol (DTT) iodoacetamide, and ammonium bicarbonate (AMBIC) were purchased from Sigma-Aldrich (St. Louis, MO); and the Zwittergent 3-12 detergent (Zwitt) was purchased from EMD Millipore (Billerica, MA).

Preparation of Immunocapture Beads. Mouse anti-human IgG antibodies were used in preparing tosylactivated beads. Dynabeads M-280 Tosylactivated ( $24 \mathrm{mg}$ or $800 \mu \mathrm{l}$ ) (Thermo Fisher Scientific, Waltham, MA) were prepared by washing with buffer A ( $0.1 \mathrm{M}$ borate buffer, $\mathrm{pH} 9.5)$ twice. A total of $480 \mu \mathrm{g}$ of antibody was mixed with the beads in buffer $\mathrm{A}$ followed by the addition of $488 \mu \mathrm{l}$ of buffer $\mathrm{C}$ ( $3 \mathrm{M}$ ammonium sulfate in buffer $\mathrm{A}$ ). The mixture was incubated on a rotator in $37^{\circ} \mathrm{C}$ overnight. The supernatant was removed after the incubation, and the mixture was incubated in $1 \mathrm{ml}$ of buffer D [PBS, pH 7.4 with $0.5 \%(\mathrm{w} / \mathrm{v})$ bovine serum albumin] on a rotator at $37^{\circ} \mathrm{C}$ for 1 hour The mixture was then washed with $1 \mathrm{ml}$ of buffer E [PBS, pH 7.4 with $0.1 \%$ (w/v) bovine serum albumin] twice and stored in $800 \mu \mathrm{l}$ of buffer $\mathrm{E}$ at $4^{\circ} \mathrm{C}$ until use (within 10 weeks). The resulting tosylactivated beads had a concentration of $0.6 \mu \mathrm{g} / \mu \mathrm{l}$ captured antibody.

Generation of Biotransformation and Control Samples of Biologics. A model therapeutic mAb $(145.0 \mathrm{kDa}, 20 \mu \mathrm{g} / \mu \mathrm{l}, 10 \mu \mathrm{l})$ was treated with $40 \mu \mathrm{l}$ of $0.1 \% \mathrm{tBHP}$ at $25^{\circ} \mathrm{C}$ overnight to introduce various protein modifications. The treated $\mathrm{mAb}$ and control $\mathrm{mAb}$ in solution $(25 \mu \mathrm{l}$ each with $100 \mu \mathrm{g}$ for $\mathrm{mAb}$ ) were spiked into $75 \mu \mathrm{l}$ of rat serum to generate a biotransformation sample and a control sample, respectively. The resulting serum samples each had $100 \mu \mathrm{g}$ of mAb with a concentration of $1 \mu \mathrm{g} / \mu \mathrm{l}$.

Sample Preparations. mAbs in rat serum were enriched by immunocapture using the tosylactivated beads, which were prepared according to the procedure described in the previous section. For each sample, $170 \mu \mathrm{l}$ of beads was loaded into a well of a 96-deep well plate followed by $200 \mu \mathrm{l}$ of buffer E and $100 \mu \mathrm{l}$ of rat serum sample. The mixture was incubated at room temperature for 1 hour on a shaker and then washed with $200 \mu \mathrm{l}$ of PBS with $0.05 \%$ Zwitt. The beads were then transferred to a fresh well using $200 \mu \mathrm{l}$ of PBS with $0.5 \mathrm{M} \mathrm{NaCl}$ and $0.05 \%$ Zwitt and washed with $200 \mu \mathrm{l}$ of $500 \mathrm{mM}$ AMBIC/0.05\% Zwitt and $200 \mu \mathrm{l}$ of $2 \mathrm{mM}$ AMBIC, and eluted with $50 \mu 1$ of $12 \mathrm{mM} \mathrm{HCl}$. The eluted fraction was transferred to a well containing $25 \mu \mathrm{l}$ of $100 \mathrm{mM}$ AMBIC and $20 \mu \mathrm{l}$ of water. Five microliters of DTT $(100 \mathrm{mM})$ was added to the mixture and incubated at $60^{\circ} \mathrm{C}$ for 60 minutes. Then $11 \mu \mathrm{l}$ of iodoacetamide $(100 \mathrm{mM})$ was added to the mixture and incubated in dark for 30-45 minutes. Trypsin was added to each sample with a 1: 25 trypsin/protein ratio (for samples with $100 \mu \mathrm{g}$ of mAb, $4 \mu \mathrm{g}$ of trypsin was added) and incubated at $37^{\circ} \mathrm{C}$ overnight. Finally, $10 \% \mathrm{FA}(1.2 \mu \mathrm{l})$ was added to make a final concentration of $0.1 \%$ FA to quench the reaction.

LC-MS Analysis. A model 1290 Infinity II LC System (Agilent Technologies, Santa Clara, CA) was connected to a TripleTOF 5600 Mass Spectrometer (AB Sciex, Framingham, MA) for all LC-MS analyses. Ten microliters of sample was injected onto a C18 column (Acquity UPLC BEH C18, $1.7 \mu \mathrm{m}$; Waters, Milford, MA) for each run. Mobile phase $\mathrm{A}$ was $\mathrm{H}_{2} \mathrm{O}$ with $0.1 \% \mathrm{FA}$, and mobile phase $\mathrm{B}$ was acetonitrile with $0.1 \%$ FA. The following gradient with a flow rate of $400 \mathrm{ng} / \mathrm{ml}$ was used (time per minute, $\%$ mobile phase B): $(0,2),(2,2),(45,45)$, $(47,90),(50,90),(51,2)$, and $(55,2)$.

To maximize the information acquired on the mass spectrometer for each sample, a full MS scan (m/z, 300-2000) was acquired followed by top 20 information-dependent acquisition (IDA) MS/MS scans (m/z, 100-1600) at positive ion mode. The parameters for CUR (curtain gas), DP (declustering potential), $\mathrm{CE}$ (collision energy), Gas1 (nebulizing gas), and Gas2 (drying gas) in full MS scan mode were $30 \mathrm{~V}, 80 \mathrm{~V}, 10 \mathrm{~V}, 55 \mathrm{psi}$, and $55 \mathrm{psi}$. The mass tolerance was $50 \mathrm{mDa}$. The source temperature was set to $450^{\circ} \mathrm{C}$, and the tray temperature was set to $22^{\circ} \mathrm{C}$. The criteria for the IDA scans were as follows: top 20 most intensive peaks with charge states from 2 to 5 and intensities greater than 50 were selected. Dynamic background subtraction was set for exclusion. Dynamic collision energy was enabled for multiply charge peptides. 


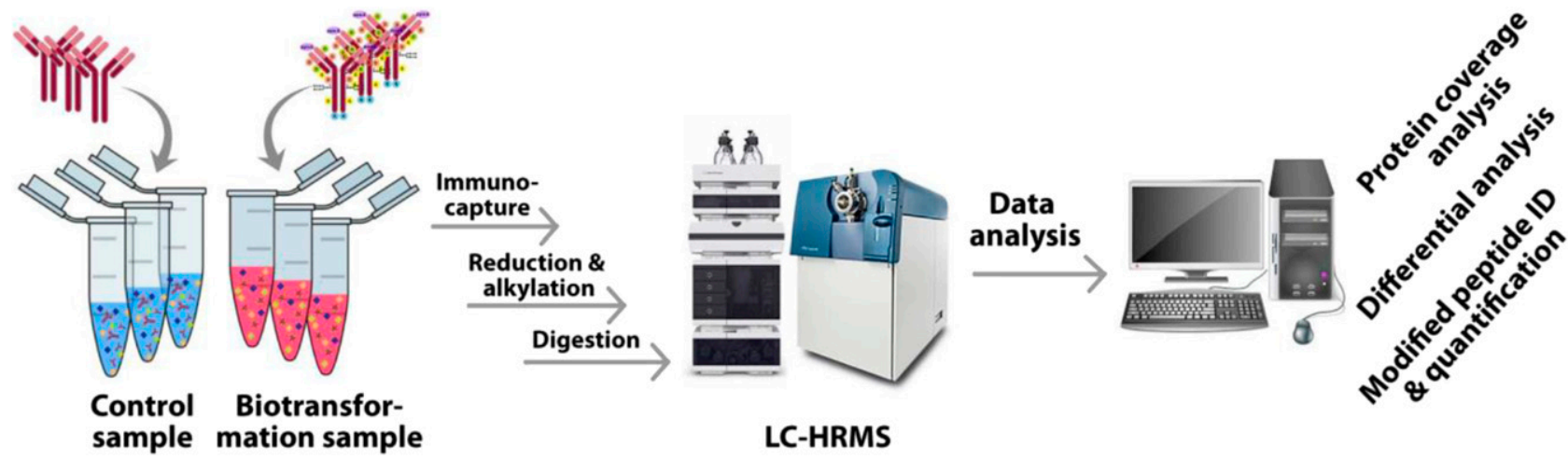

Fig. 1. Workflow of LC-HRMS-based differential analysis for detecting biotransformation-mediated modifications. ID, identification.

Data Processing. A customized version of PEAKS version 7.5 (Bioinformatics Solutions Inc.) was used for peptide mapping, and PTM (Bioinformatics Solutions Inc.) was used for searching and differential analysis (label-free quantification). The following parameters were used for peptide mapping against the tocilizumab database: parent mass error tolerance of $50 \mathrm{ppm}$, fragment mass error tolerance of $1.0 \mathrm{Da}$, and up to three missed cleavages were allowed. NQ and oxidation were set as variable modifications. Carbamidomethylation was set as a fixed modification. All 53 common and 420 uncommon PTMs were included for PTM searching. Up to three PTMs were allowed for each peptide. The false discovery rate was set at $1 \%$. Each data file was normalized to the total ion count before differential analysis. One of the control samples was used as a reference sample for normalization to compare the ratio for each peptide in different samples. After database searching and PTM analysis, the data files were processed with differential analysis. The resulting heat map represents the $\log 2$ ratio to the average peak area across different samples. Different filtering criteria were used for data interpretation

\section{Results}

A Bottom-Up Proteomics-Based Differential Analysis Workflow. In the current study, a bottom-up, proteomics-based differential analysis strategy was developed and evaluated for untargeted detection and characterization of global biotransformation products on a therapeutic $\mathrm{mAb}$ (Fig. 1). In this analysis, a biotransformation sample and a corresponding control sample are compared. The biotransformation sample can either be from an in vitro incubation of a therapeutic protein or collected from an animal after dosing a therapeutic protein. The control was either a preincubation or a predosing sample. Immunocapture and/or other methods were employed to recover and enrich the therapeutic protein and its modification products from the biologic matrix. The recovered proteins were further reduced, alkylated, and digested by trypsin, followed by peptide analyses via LC-HRMS. The resulting full-scan MS and MS/MS datasets of the biotransformation and control samples, which could be acquired by a data-dependent or data-independent method, were subjected to peptide mapping, modification analysis, and differential analysis using customized proteomics data-processing software. Peptide mapping was achieved by comparing the acquired MS2 spectra with theoretically calculated fragment ion $\mathrm{m} / \mathrm{z}$ values from the $\mathrm{mAb}$ amino acid residue sequence. Peptide modification analysis was achieved by searching the mass differences of known modifications and compared with the acquired MS2 spectrum. Differential analysis was performed by comparing the peak area of extracted ion chromatograms of detected features from the biotransformation and control samples in triplicate. Peptide mapping coverage was determined to ensure the quality of protein hydrolysis and peptide mapping processes. Peptides modified via biotransformation, which were either absent in the control sample or had significantly higher levels in the test sample, were determined using differential analysis.
Furthermore, sequences of modified peptides were elucidated automatically using the software or by manual interpretation for uncommon peptide modifications. In the current study, simplified biotransformation and control samples of a human mAb were generated to test the effectiveness of the workflow (Fig. 1).

The Coverage of Peptide Mapping and Identification of Protein Modifications. The sequence coverage and peptide modifications detected in the light chain and heavy chain of the control mAb sample are presented in Supplemental Fig. 1. The sequence coverage was calculated using the number of amino acid residues detected divided by the total number of amino acid residues in the known mAb sequence. By using the untargeted LC-HRMS approach, 93\% of the light chain and $92 \%$ of the heavy chain were covered when the false discovery rate was set at $1 \%$. The result indicated that the quality of protein processes and LC/HRMS analysis are good. Sixty and 129 unique peptides, respectively, were detected for the light chain and heavy chain of the mAb recovered from the biotransformation sample. Common modifications, such as carbamidomethylation, NQ, and oxidation, were labeled in the figures. Additionally, less common protein modifications, such as sulfation and pyro-Glu from Glu (Supplemental Fig. 1; Table 1), could

TABLE 1

Partial list of PTMs detected by PEAKS PTM algorithm

\begin{tabular}{lrccc}
\hline \multicolumn{1}{c}{ Name } & \#PSM & \multicolumn{1}{c}{$\Delta$ Mass } & Position & $-10 \lg$ \\
\hline Carbamidomethyl & 1638 & 57.02 & C & 200 \\
Carbamidomethyl & 1407 & 57.02 & DEHK, N-terminal & 132.5 \\
Deamidation & 964 & 0.98 & NQ & 200 \\
Oxidation & 223 & 15.99 & M & 155.8 \\
Dihydroxy & 143 & 31.99 & CFKPRW & 126.5 \\
Oxidation & 129 & 15.99 & HW & 122.7 \\
Ubiquitin & 80 & 114 & CKST & 84.31 \\
Ammonia loss & 59 & -17.03 & N & 130.8 \\
Ammonium & 56 & 17.03 & DE, C-terminal & 53.12 \\
Hydroxylation & 39 & 15.99 & DKNPY & 72.49 \\
Pyro-glu from Q & 34 & -17.03 & N-terminal & 105.1 \\
Dehydration & 33 & -18.01 & DSTY, C-terminal & 87.02 \\
DehydroalaC & 26 & -33.99 & C & 104.9 \\
Trifluoro & 23 & 53.97 & L & 43.29 \\
Cysteic acid & 22 & 47.98 & C & 83.85 \\
Amidation & 19 & -0.98 & C-terminal & 56.34 \\
Dethiomethyl & 18 & -48 & M & 43.77 \\
Methyl ester & 15 & 14.02 & DE, C-terminal & 38.11 \\
Methylation & 14 & 14.02 & S & 42.68 \\
Carbamylation & 13 & 43.01 & N-terminal & 35.57 \\
Sulfation & 13 & 79.96 & ST & 133.3 \\
Carboxylation & 12 & 43.99 & DK & 55.7 \\
Pyro-cmC & 10 & 39.99 & N-terminal & 87.79 \\
Carbamidomethyl DTT & 10 & 209 & C & 62.44 \\
\hline
\end{tabular}

$\triangle$ Mass, change in mass of each modification; \#PSM, corresponding peptide spectrum match. 


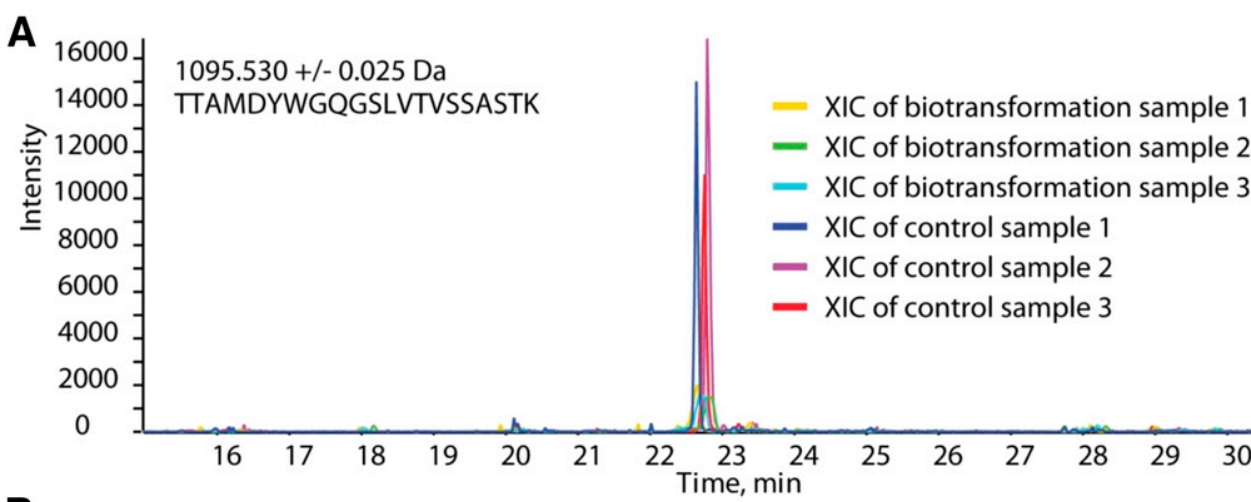

B

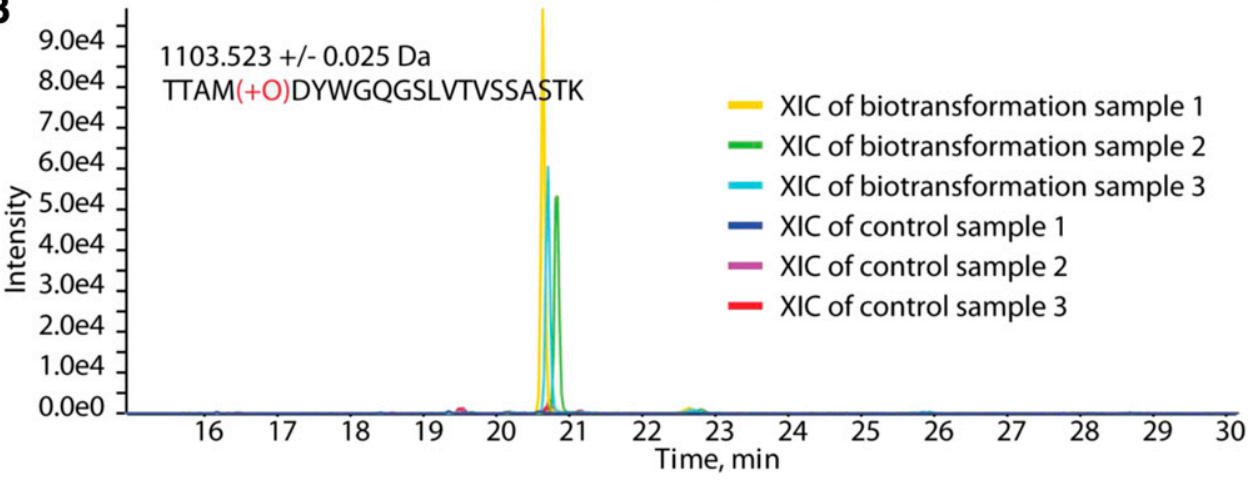

Fig. 2. Extracted ion chromatogram (XIC) that show significant differences between biotransformed and control groups of sample. (A) XIC of $\mathrm{m} / \mathrm{z} 1095.530$ for both control and biotransformed samples. (B) XIC of $\mathrm{m} / \mathrm{z}$ 1103.523 for for both control and biotransformed samples. be found via data processing using the customized software. In total, 37 types of modifications were determined and characterized by the PEAKS PTM search algorithm (Supplemental Table 1). The corresponding peptide spectrum match, change in mass of each modification, position, and $-10 \log \mathrm{P}$ values are listed in Table 1. Carbamidomethyl, NQ, oxidation, and dihydroxy were among the common popular modifications; other less common ones, such as point mutation of single amino acid, sulfation, and carboxylation, were also detected (Supplemental Fig. 1).

Identification of Modified Peptides Derived by Biotransformation. Modified peptides derived from biotransformation were determined by differential analysis (Fig. 2). These peptides were either only present or had a significantly higher level in the biotransformation sample. Table 2 summarized a partial list of these peptides with significance values $(-10 \log \mathrm{P})$ above 5 . Most of these peptides were associated with methionine oxidation. In the sample profile and group profile columns, the left panel represented the biotransformation samples, whereas the right panel represented the control samples. All the unoxidized peptides (rows 1, 3, 5, and 7) had much higher intensities in the control samples, whereas all the oxidized peptides (rows 2, 4, 6, and 8) had much higher intensities in the biotransformation samples. Some data points were not shown in the sample profile because the corresponding peptide intensity was below detection.

As an example, the extracted ion chromatogram of the doubly charged unmodified and oxidized forms of a peptide

TABLE 2

Partial list of significantly changed peptides by differential analysis

In the Sample Profile and Group Profile columns, the left panel represented the biotransformed samples and the right panel represented the control samples. In the Sample Profile and Group Profile

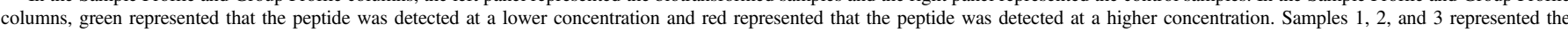
biotransformation samples, and samples 4,5 , and 6 represented the control samples.

\begin{tabular}{|c|c|c|c|c|c|c|c|}
\hline Peptide & $\begin{array}{l}\text { Significance } \\
(-10 \lg P)\end{array}$ & $\mathrm{m} / \mathrm{z}$ & $z$ & $\begin{array}{l}\text { Avgerage } \\
\text { Area }\end{array}$ & Sample Profile & Group Profile & PTM \\
\hline DTLMISR & 29.63 & 418.2209 & 2 & $1.40 \mathrm{E}+04$ & & & \\
\hline DTLM(+15.99)ISR & 18.98 & 426.2198 & 2 & $2.70 \mathrm{E}+04$ & & & Oxidation (M) \\
\hline SVMHEALHNHYTQK & 16.39 & 565.6129 & 3 & 660.4 & & & \\
\hline SVM(+15.99)HEALHNHYTQK & 7.72 & 428.4561 & 4 & 180.6 & & & Oxidation (M) \\
\hline SC(+57.02)SVMHEALHNHYTQK & 17.57 & 486.2236 & 4 & 513.1 & & & Carbamidomethylation \\
\hline $\begin{array}{l}\text { SC(+57.02)SVM(+15.99) } \\
\text { HEALHNHYTQK }\end{array}$ & 12.71 & 490.2209 & 4 & 238.2 & & & $\begin{array}{c}\text { Carbamidomethylation Oxidation } \\
\text { (M) }\end{array}$ \\
\hline TTAMDYWGQGSLVTVSSASTK & 12.94 & 730.6826 & 3 & 666.4 & & & \\
\hline $\begin{array}{l}\text { TTAM(+15.99) } \\
\text { DYWGQGSLVTVSSASTK }\end{array}$ & 17.17 & 1103.523 & 2 & $6.20 \mathrm{E}+03$ & & & Oxidation (M) \\
\hline
\end{tabular}




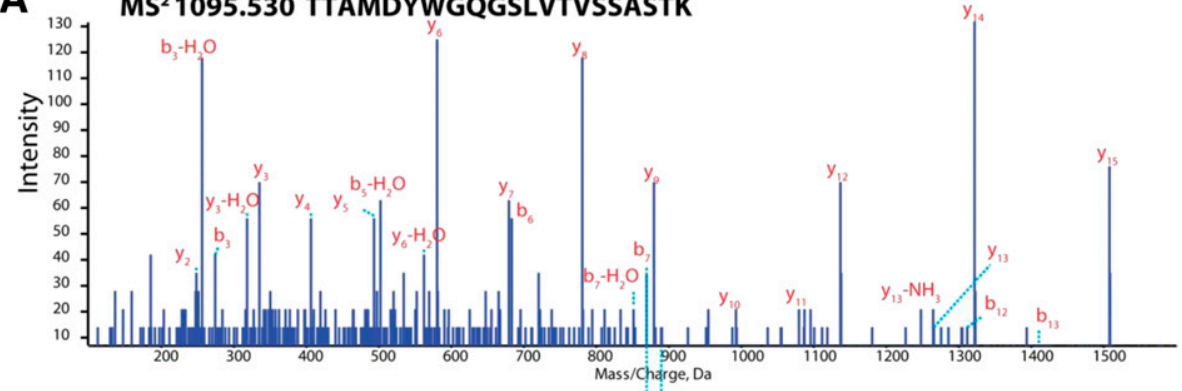

B

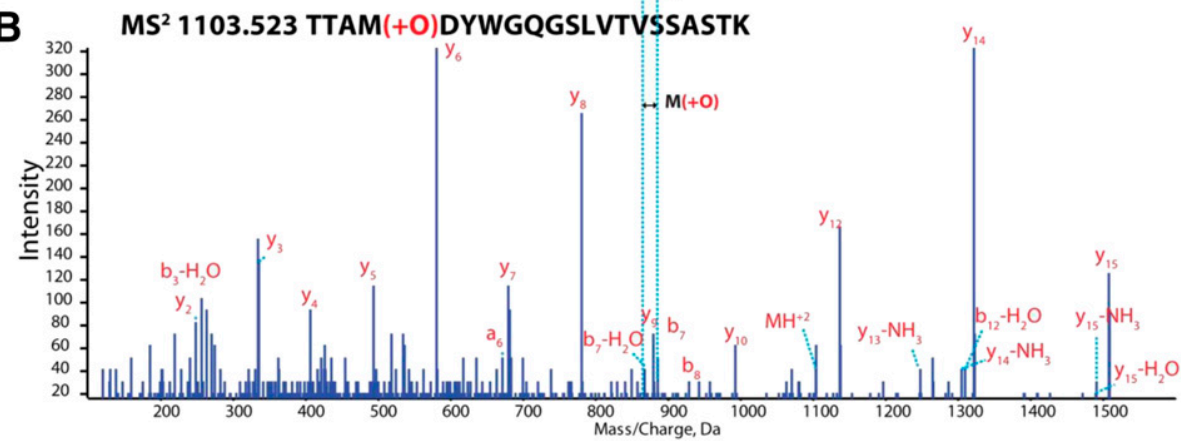

Fig. 3. Representative MS/MS spectra of $\mathrm{m} / \mathrm{z}$ 1095.530 (A) and $\mathrm{m} / \mathrm{z} 1103.523$ (B), $z=2$ for both ions. Peptide sequences and fragment ions were annotated on the spectra.

(TTAMDYWGQGSLVTVSSASTK) are shown in Fig. 2. A high abundance of the unmodified peptide was observed in all three of the control samples with intensities above $1 \mathrm{E} 4$, whereas the signals were barely detected in the biotransformation sample (Fig. 2A). On the other hand, a high abundance of the oxidized form of this peptide was observed in the biotransformation sample, but barely any signal was detected in the control sample (Fig. 2B). A 2-minute shift in retention time was observed between these peptide forms, which was associated with the oxidation of methionine that increased the hydrophilicity of the peptide.

The corresponding MS2 spectra of these peptides (Fig. 3) were acquired by the IDA scans during the elution of the corresponding peptides. For both peptides, y 2 to y 15 ions (except for the y11 ion of the oxidized form) and some of the $\mathrm{b}$ ions were detected with high abundance. Based on the fragment ion information, the sequence of these peptides could be confidently assigned by the database-searching algorithm of PEAKS. By comparing the spectra of unmodified (Fig. 3A) and oxidized (Fig. 3B) forms, the mass difference between methionine and oxidized methionine $(+15.99)$ was observed in the b7 ions (shown in dotted blue lines) as well as some other b ions. The mass difference in the MS2 spectra allows the database-searching algorithm as well as the PTM analysis algorithm to detect the type and location of potential modifications existing in each peptide.

Quantitative Estimation of Modified Peptides Derived by Biotransformation. The percentage of modified peptides presented in each sample was calculated and summarized in Fig. 4 (common modifications of heavy chain) and Table 3 (other modifications). By examining the oxidation profiles, a large percentage of methionine residues in the biotransformed samples have been oxidized (Fig. 4A). The highly oxidized $(>95 \%)$ residues included M70o, M254o, and M430o of the heavy chain. About 50\% of the M106o had also been oxidized. On the other hand, most of the methionine residues in the control samples remained unmodified (Fig. 4B). Only 5\%-10\% of the M106 was oxidized in the control sample, which could be modified during the storage or sample preparation process. Additionally, the disappearance of unmodified peptides in the biotransformation and control samples was quantitatively estimated. As shown in Fig. 4, some unmodified peptides, such as M106o and M254o, were absent and some, such as $\mathrm{M} 70 \mathrm{o}$, remained a small percentage after biotransformation. Furthermore, some unmodified peptides were absent in both biotransformation and control samples, such as carbamidomethylated residues $\mathrm{C} 228 \mathrm{c}$ and $\mathrm{C} 263 \mathrm{c}$, due to sample processing rather than biotransformation.

Summary of Biotransformation-Derived Protein Modifications. The modified percentages of other types of modifications, including NQ, carbamidomethylation, and dimethylation, were consistent in both sample groups (Fig. 4, C and D; Table 3). The sources of these modifications were mostly from production, storage, or sample preparation, which were not directly related to tBHP treatment.

Figure 5 summarized the results of differential analysis with a significance $(-10 \log \mathrm{P})$ of at least 15 and fold changes of at least 2-fold. Peptides confidently detected in at least three samples (Fig. 5A) and peptides confidently detected in all six samples (Fig. 5B) were listed. Figure 5C represents the relationship among these datasets. As some peptides were undetectable in the control or biotransformed group, features detected in both sets $(n=3)$ samples (in control group or biotransformed group), and all six samples were monitored.

\section{Discussion}

A rapid and sensitive LC-HRMS-based strategy was developed in this study to characterize the biotransformation-mediated modifications in an artificially oxidized mAb model system. Due to the similarity in property- and sample-handling procedures, this approach is also suitable for other classes of therapeutic biologics, such as therapeutic peptides, $\mathrm{ADC}$, and bifunctional biologics.

Most of the previous studies on biotransformation focused on one particular type of modification, such as oxidation (Wang et al., 2011) or N-terminal glutamate-to-pyroglutamate conversion (Liu et al., 2011). Although these in vivo studies allow in-depth structure elucidation, they are not as informative as a high-throughput study to screen all modifications in one run. By using the untargeted LC-HRMS-based 


\section{Oxidation Profile}
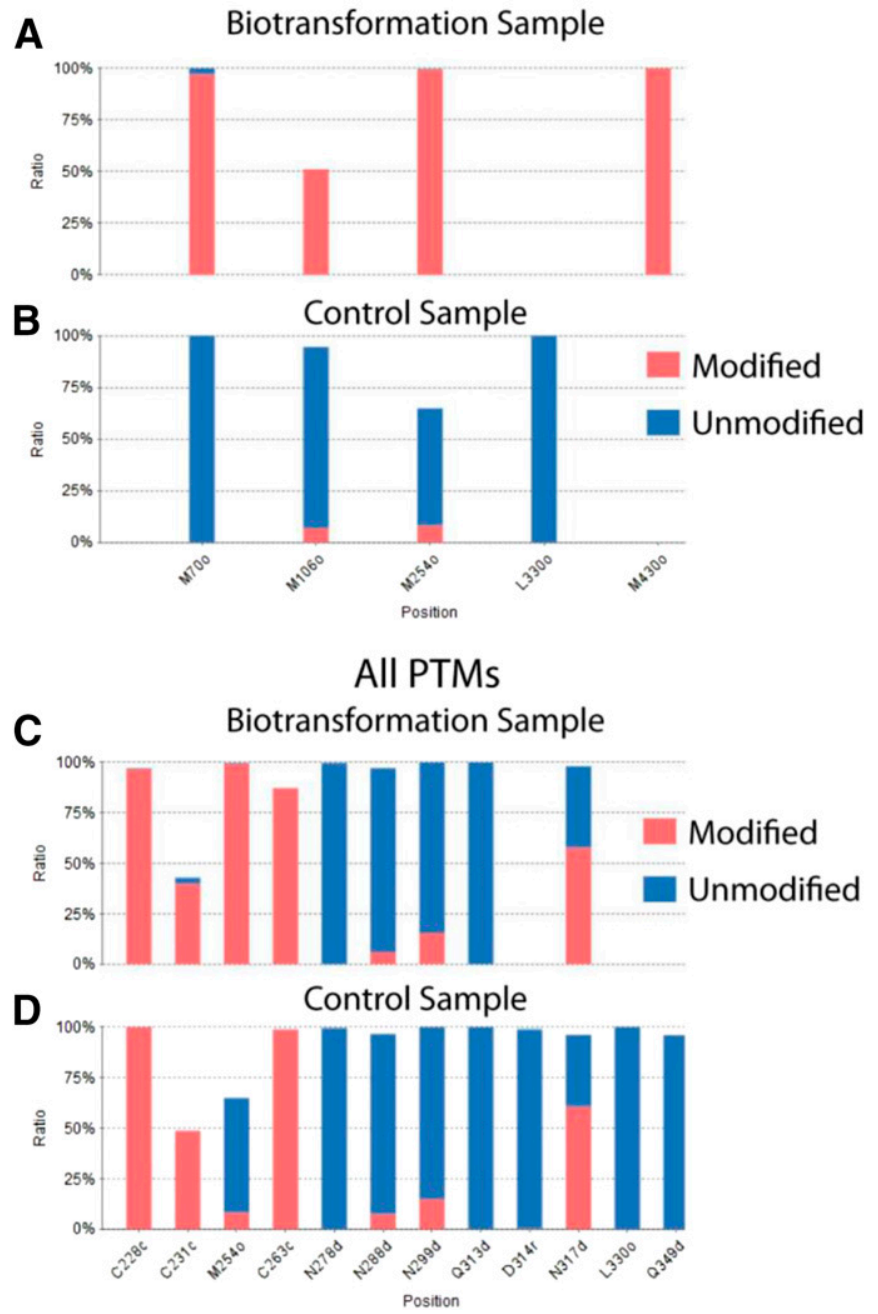

Fig. 4. Partial PTM profiles of biotransformation (A and C) and control (B and D) samples. (A and B) compared the oxidation profiles, and (C and D) compared all modifications. The column in red represented the modified peptides and the column in blue represented unmodified peptides. In the $x$-axis, the capital letter represented corresponding amino acid residues, the number represented the positions of these modifications, and the last letter represented the types of modifications, as follows: d, deamination; c, carbamidomethylation; o, oxidation. The $y$-axis represented the percentage of unmodified or modified peptides related to the sum of this peptide with all modifications. (C) D314r, $\mathrm{L} 330 \mathrm{o}$, and Q349d were not detected in the biotransformation sample.

approach, different modifications can be detected in a high-throughput fashion to generate valuable information, whereas the sources of modifications (storage, sample preparation, or treatment) potentially could be unveiled by differential analysis. The uniqueness of this workflow is mainly presented in the following two aspects: 1) instead of focusing on one modification, all modifications can be examined at one time with the use of bioinformatic tools; and 2) by comparing with control samples, the shared modifications were filtered away, and only the modifications of interest were retained and thus reported.

The conserved methionine residues (M254 and M430), located in the FnRn binding site and playing important roles in FcRn binding, were found to be oxidized in the biotransformation samples. This result is consistent with that of a previous report (Wang et al., 2011) that oxidizing these two methionine residues at a high level could significantly reduce the serum circulation half-life of mAbs.

Due to the large size of therapeutic biologics and the large number of potential modification sites, there exists a large number of low-level modifications widely present in all samples. Most of these modifications, which are not directly related to the biotransformation process, are introduced during production, storage, and sample preparations. By performing database searching and PTM analysis using bioinformatic tools, we observed a relatively high level of basal modifications in the control samples. Moreover, most of the modification types and modified peptides are shared by the control and biotransformation samples. Some random modifications were observed in only one replicate of either the control or biotransformation samples. Therefore, it is difficult to pick out the biotransformation-mediated modifications that were induced by the tBHP treatment in this case without using differential analysis. Furthermore, if conventional LBAs are used, these modifications can impact drug detection and are convoluted with the actual biotransformation-mediated modifications results, leading to overestimation of the degree of biotransformation within biologics. In contrast, LC-HRMS-based differential analysis enables direct analysis of the biotransformation-mediated modifications without including unspecific modifications, which generates more accurate results and saves time and effort from not analyzing irrelevant data.

Moreover, the possible source of modifications can be determined by this approach. If the modifications are induced by a certain treatment, significant differences can be observed between biotransformed and control samples; if the modifications are related to storage, differences can be observed between old and fresh samples; if the modifications are associated with sample preparation, differences will be universally presented in all sample groups.

It is worth pointing out that there could be discrepancies during the immune-based enrichment process between control and biotransformation samples, as the targeted epitope in the biotransformed sample could be altered during tBHP treatment. Other enrichment methods will be compared in future studies. Also, the quantitative analysis of stoichiometry of modification was based on the peak area ratio of the modified and unmodified peptides. However, there could be differences in

TABLE 3

Summary of peptides that underwent other significant biotransformation

The mass difference of each PTM was shown in the peptide sequence: deamidation $(+0.98)$, carbamidomethylation $(+57.02)$, and dimethylation $(+28.03)$.

\begin{tabular}{lccc}
\hline \multirow{1}{*}{ Native Peptides } & & \multicolumn{2}{c}{$\%$ Modified } \\
\cline { 2 - 4 } & Modified Peptides & Biotransformed & Control \\
\hline TVLHQDWLNGK & & 45.5 & 36.6 \\
VVSVLTVLHQDWLNGK & TVLHQDWLN(+0.98)GK & 76.3 & 39.6 \\
C $(+57.02)$ PAPELLGGPSVFLFPPKPK & C (+57.02)(+28.03)PAPELLGGPSVFLFPPKPK & 55.5 & 44.3 \\
EEQYNSTYR & EEQYN(+0.98)STYR & 42 & 10.5 \\
FNWYVDGVEVHNAK & FNWYVDGVEVHN(+0.98)AK & 3.3 & 4.8 \\
HQDWLNGK & HQDWLN(+0.98)GK & 64 & 51 \\
IC(+57.02)NVNHKPSNTK & IC(+57.02)NVN(+0.98)HKPSNTK & 6.4 & 0 \\
\hline
\end{tabular}


A Peptides detected in 3 samples
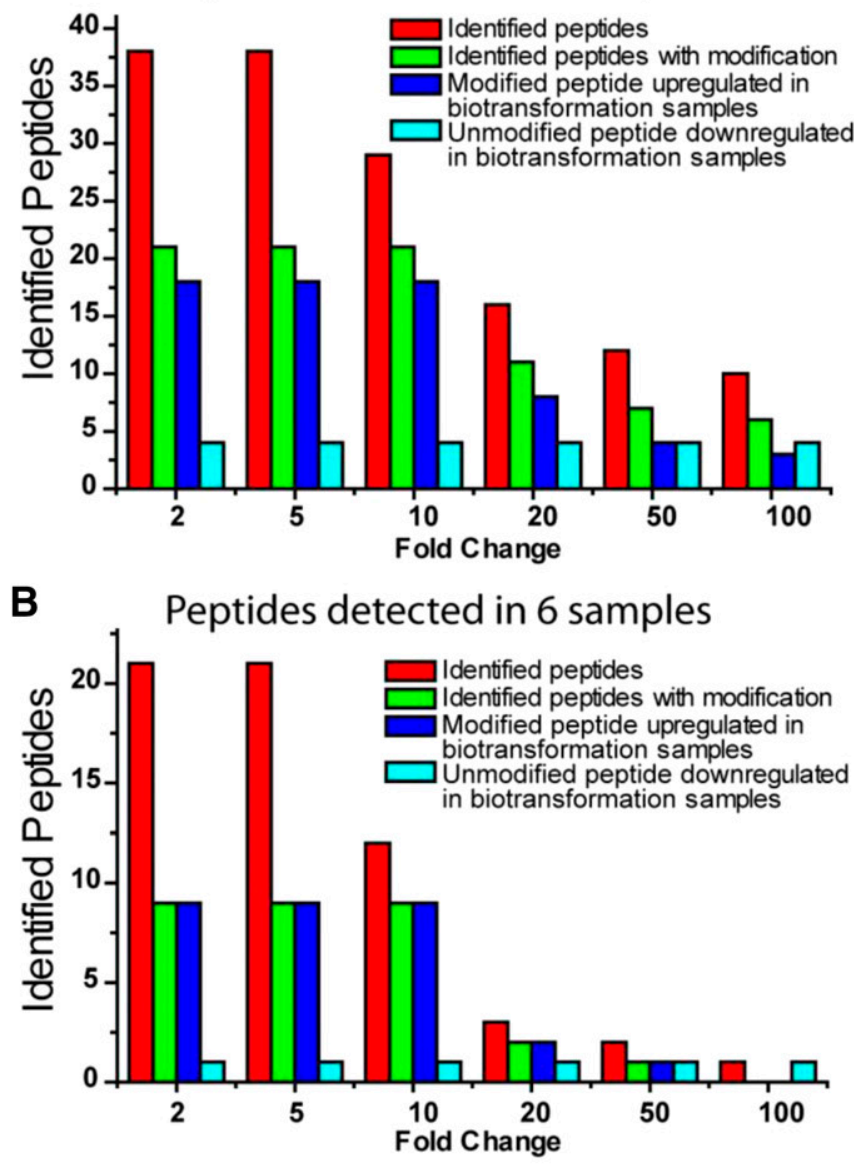

C

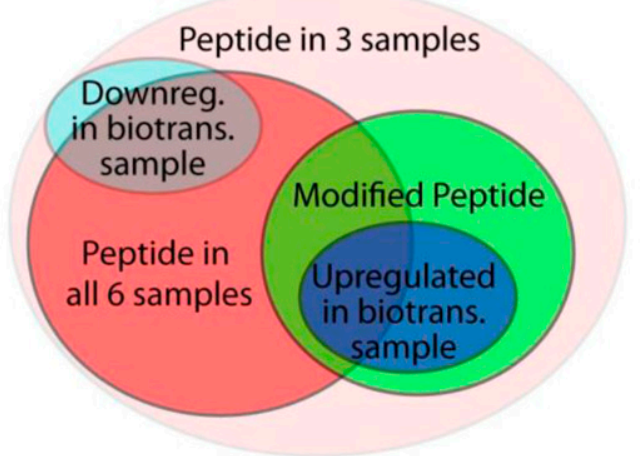

Fig. 5. Features detected by differential analysis with a significance $(-10 \log \mathrm{P})$ of 15 in at least three samples (A) and all six samples (B), and a Venn diagram (C) representing the relationships among these datasets. The $x$-axis represents fold changes of $2,5,10,20,50$, and 100 . biotrans., biotransformation.

ionization efficiency between the modified and unmodified peptide. To precisely determine the actual stoichiometry, an internal standard-based quantification approach should be used in addition to the method developed in this study.

In summary, a novel approach was developed to systematically study the universal biotransformation of therapeutic biologics using LC-HRMS-based untargeted differential analysis. With this approach, sequence coverage of the human $\mathrm{mAb}$ was above $92 \%$ and 37 types of modifications were identified (all point mutations were treated as one type of modification). As many as 38 peptides were significantly changed, up to 21 of them have modifications and up to 18 of the modified peptides with modifications increased in the biotransformed samples compared with the control samples. In this method, different modifications could be rapidly detected and the sources of modification could be potentially unveiled by differential analysis as coexisting modifications occurring in both samples are filtered out, revealing the modifications specifically due to biotransformation. This method will be used for studying the in vivo biotransformation of test proteins in a future study.

Due to similarity in property and sample-handling procedures, this approach is also suitable for other classes of therapeutic biologics, such as therapeutic peptides, ADCs, and bifunctional biologics. This new analytical strategy can be effectively applied to study biotransformation-mediated protein modifications in the process of drug discovery and development.

\section{Acknowledgments}

We thank Bioinformatics Solutions Inc. for technical support on the PEAKS software. M.Z. thanks Pharmaceutical Candidate Optimization, Bristol-Myers Squibb Research, and Development for supporting this study.

\section{Authorship Contributions}

Participated in research design: Yao and Zhu.

Conducted experiments: Yao and Zhao.

Performed data analysis: Yao, Chen, Mehl, and Zhu.

Wrote or contributed to the writing of the manuscript: Yao, Chen, Zhao, Mehl, $\mathrm{Li}$, and Zhu.

\section{References}

An B, Zhang M, and Qu J (2014) Toward sensitive and accurate analysis of antibody biotherapeutics by liquid chromatography coupled with mass spectrometry. Drug Metab Dispos 42:1858-1866. Bults P, Bischoff R, Bakker H, Gietema JA, and van de Merbel NC (2016) LC-MS/MS-based monitoring of in vivo protein biotransformation: quantitative determination of trastuzumab and its deamidation products in human plasma. Anal Chem 88:1871-1877.

Cai B, Pan H, and Flynn GC (2011) C-terminal lysine processing of human immunoglobulin G2 heavy chain in vivo. Biotechnol Bioeng 108:404-412.

Cheloha RW, Maeda A, Dean T, Gardella TJ, and Gellman SH (2014) Backbone modification of a polypeptide drug alters duration of action in vivo. Nat Biotechnol 32:653-655.

Domon B and Aebersold R (2006) Mass spectrometry and protein analysis. Science 312:212-217.

Ewles M and Goodwin L (2011) Bioanalytical approaches to analyzing peptides and proteins by LC--MS/MS. Bioanalysis 3:1379-1397.

Hall MP (2014) Biotransformation and in vivo stability of protein biotherapeutics: impact on candidate selection and pharmacokinetic profiling. Drug Metab Dispos 42:1873-1880.

Hamuro LL and Kishnani NS (2012) Metabolism of biologics: biotherapeutic proteins. Bioanalysis 4:189-195.

Han X, He L, Xin L, Shan B, and Ma B (2011) PeaksPTM: mass spectrometry-based identification of peptides with unspecified modifications. J Proteome Res 10:2930-2936.

Kang L, Camacho RC, Li W, D'Aquino K, You S, Chuo V, Weng N, and Jian W (2017) Simultaneous catabolite identification and quantitation of large therapeutic protein at the intact level by immunoaffinity capture liquid chromatography-high-resolution mass spectrometry. Anal Chem 89:6065-6075.

Katsila T, Siskos AP, and Tamvakopoulos C (2012) Peptide and protein drugs: the study of their metabolism and catabolism by mass spectrometry. Mass Spectrom Rev 31:110-133.

Kullolli M, Rock DA, and Ma J (2017) Immuno-affinity capture followed by TMPP N-terminus tagging to study catabolism of therapeutic proteins. J Proteome Res 16:911-919.

Liu YD, Goetze AM, Bass RB, and Flynn GC (2011) N-terminal glutamate to pyroglutamate conversion in vivo for human IgG2 antibodies. J Biol Chem 286:11211-11217.

Liu YD, van Enk JZ, and Flynn GC (2009) Human antibody Fc deamidation in vivo. Biologicals 37:313-322.

Prueksaritanont $\mathrm{T}$ and Tang $\mathrm{C}$ (2012) ADME of biologics-what have we learned from small molecules? AAPS J 14:410-419.

Ruan Q, Ji QC, Arnold ME, Humphreys WG, and Zhu M (2011) Strategy and its implications of protein bioanalysis utilizing high-resolution mass spectrometric detection of intact protein. Anal Chem 83:8937-8944.

Waldmann TA (2003) Immunotherapy: past, present and future. Nat Med 9:269-277.

Walles M, Rudolph B, Wolf T, Bourgailh J, Suetterlin M, Moenius T, Peraus G, Heudi O, Elbast W, Lanshoeft C, et al. (2016) New insights in tissue distribution, metabolism, and excretion of $[3 \mathrm{H}]$-labeled antibody maytansinoid conjugates in female tumor-bearing nude rats. Drug Metab Dispos 44:897-910.

Wang W, Vlasak J, Li Y, Pristatsky P, Fang Y, Pittman T, Roman J, Wang Y, Prueksaritanont T, and Ionescu R (2011) Impact of methionine oxidation in human IgG1 Fc on serum half-life of monoclonal antibodies. Mol Immunol 48:860-866.

$\mathrm{Xu} \mathrm{X}$ and Vugmeyster Y (2012) Challenges and opportunities in absorption, distribution, metabolism, and excretion studies of therapeutic biologics. AAPS J 14:781-791.

Zhang D, Yu SF, Ma Y, Xu K, Dragovich PS, Pillow TH, Liu L, Del Rosario G, He J, Pei Z, et al. (2016) Chemical structure and concentration of intratumor catabolites determine efficacy of antibody drug conjugates. Drug Metab Dispos 44:1517-1523.

Zhao L, Ren TH, and Wang DD (2012) Clinical pharmacology considerations in biologics development. Acta Pharmacol Sin 33:1339-1347.

Address correspondence to: Dr. Mingshe Zhu, MassDefect Technologies, PO Box 172, Rocky Hill, NJ 08553. E-mail: mingshe.zhu@yahoo.com; or Dr. Lingjun Li, School of Pharmacy, University of Wisconsin-Madison, 777 Highland Avenue, Madison, WI 53705-2222. E-mail: lingjun.li@wisc.edu 